\title{
Emprego do Laser de baixa intensidade no pós-operatório de exodontia de terceiros molares
}

Use of low level laser therapy in the postoperative of exodontia of third molars

Uso de láser de bajo nivel en el postoperatorio de extracción de terceros molares

Rodrigo Hartmann ATUÁ ${ }^{1}$

Key Fabiano Souza PEREIRA ${ }^{2}$

Ellen Cristina GAETTI JARDIM ${ }^{2}$ Edilson José ZAFALON ${ }^{2}$

Evelyn de Freitas BOSCAINE ${ }^{1}$

Julio Cesar Leite da SILVA ${ }^{2}$

${ }^{1}$ Alunos do Programa de Pós-Graduação Mestrado "Strictu Sensu" da Faculdade de Odontologia da Universidade Federal de Mato Grosso do Sul (FAODO) Universidade Federal do Mato Grosso do Sul (UFMS) 79070-900 Campo Grande - MS, Brasil

${ }^{2}$ Professores Doutores da Faculdade de Odontologia da Universidade Federal de Mato Grosso do Sul (FAODO) Universidade Federal do Mato Grosso do Sul (UFMS) 79070-900 Campo Grande-MS, Brasil

\section{Resumo}

Esse estudo split mouth, duplo cego, randomizado teve como proposta avaliar o uso do Laser de baixa intensidade no pósoperatório de pacientes submetidos a exodontias de terceiros molares inclusos. Os participantes receberam a mesma terapêutica farmacológica e técnica cirúrgica. Dez voluntários, com idade entre 16 e 26 anos, sem doenças locais ou sistêmicas, com terceiros molares inferiores inclusos bilateralmente, com disposição similar e extração indicada, foram submetidos a cirurgias para sua remoção. Em um grupo foi utilizado Laser vermelho 660nm após anestesia e sutura, com uma dose de $3 \mathrm{~J}$ por 30 segundos nas faces vestibular e lingual da região operada, e no pós-operatório mediato entre 24 e $72 \mathrm{~h}$. $\mathrm{O}$ Laser infravermelho $808 \mathrm{~nm}$, com uma dose de $3 \mathrm{~J}$ por 30 segundos também foi utilizado nos linfonodos logo após a cirurgia e no pós-operatório entre 24 e 72 horas, já no grupo controle o Laser não foi aplicado. Após análise estatística, realizada por meio do teste t-student pareado, os resultados mostraram diferença significativa entre os lados experimentais, mostrando melhora na dor pós-operatória, na diminuição de edema e na limitação da abertura bucal, no lado onde o Laser foi aplicado. O Laser de baixa intensidade mostrou-se eficiente no pós-operatório de exodontia de terceiros molares inferiores inclusos.

Descritores: Dor; Edema; Terapia com Luz de Baixa Intensidade; Dente Serotino.

\section{Abstract}

This double-blind, split-mouth, randomized study aimed to evaluate the use of low-level laser in the postoperative of patients undergoing extraction of third molars. Participants received the same pharmacological therapy and surgical technique. Ten volunteers, aged between 16 and 26 years, with no local or systemic diseases, lower third molars included bilaterally, similar disposition and indicated extraction, underwent surgery for their removal. In a group, a 660nm red laser was used after anesthesia and suture, with a dose of $3 \mathrm{~J}$ for 30 seconds on the buccal and lingual surfaces of the operated region, and in the mediate postoperative period between 24 and 72 hours. The $808 \mathrm{~nm}$ infrared laser, with a dose of $3 \mathrm{~J}$ for 30 seconds, was also used in the lymph nodes immediately after surgery and in the postoperative period between 24 and 72 hours, in the control group the laser was not applied. After statistical analysis, performed using the paired t-student test, the results showed a significant difference between the experimental sides. There were improvement in postoperative pain, reduction of edema and in the limitation of the mouth opening, on the side where the laser was applied. The low-level laser proved to be efficient in the post-operative extraction of lower third molars.

Descriptors: Pain; Edema; Low-Level Light Therapy; Molar, Third.

\section{Resumen}

Este estudio split mouth, doble ciego, aleatorizado tuvo como objetivo evaluar el uso de láser de bajo nivel en el período postoperatorio de pacientes sometidos a extracción de terceros molares. Los participantes recibieron la misma terapia farmacológica y técnica quirúrgica. Diez voluntarios, de edades comprendidas entre 16 y 26 años, sin enfermedades locales o sistémicas, con terceros molares inferiores incluidos bilateralmente, con disposición similar y extracción indicada, se sometieron a cirugía para su extracción. En un grupo, se usó un láser rojo de $660 \mathrm{~nm}$ después de la anestesia y la sutura, con una dosis de 3J durante 30 segundos en las superficies vestibular y lingual de la región operada, y en el período postoperatorio medio entre 24 y 72 horas. El láser infrarrojo de $808 \mathrm{~nm}$, con una dosis de $3 \mathrm{~J}$ durante 30 segundos, también se usó en los ganglios linfáticos inmediatamente después de la cirugía y en el período postoperatorio entre 24 y 72 horas, en el grupo control no se aplicó el láser. Después del análisis estadístico, realizado utilizando la prueba de t-student emparejada, los resultados mostraron una diferencia significativa entre los lados experimentales, mostrando una mejora en el dolor postoperatorio, en la reducción del edema y en la limitación de la apertura de la boca, en el lado donde se aplicó el láser. . El láser de baja intensidad demostró ser eficiente en la extracción postoperatoria de terceros molares inferiores.

Descriptores: Dolor; Edema; Terapia por Luz de Baja Intensidad; Tercer Molar.

\section{INTRODUÇÃO}

Em decorrência da não erupção espontânea dos terceiros molares, a população jovem pode apresentar problemas nessa região da cavidade bucal como, dor, edema, infecção, trismo e dificuldade de higiene oral. O grande número de casos de dentes inclusos pode ser originado da falta de espaço para erupção ou simplesmente o seu mau posicionamento". Portanto, a exodontia de terceiros molares inferiores inclusos é um dos procedimentos mais comuns realizados por cirurgiões-dentistas e algumas importantes complicações cirúrgicas podem ocorrer no pós-operatório do procedimento ${ }^{2}$. Entre elas, destacam-se a dor pós-operatória (DPO), o edema e a limitação da abertura bucal denominado como trismo, em virtude do trauma cirúrgico ${ }^{3,4}$.

Apesar das exodontias de terceiros molares estarem se tornando cada vez mais rotineira ela apresenta grandes desafios a serem superados. O fato recai eventualmente em causar trauma cirúrgico significativo e desencadear uma resposta imunopatológica inata de defesa, a inflamação ${ }^{5}$. O trauma 
envolvendo ossos e tecidos moles em face que é intensamente vascularizada, e os tecidos moles constituídos por tecido conjuntivo frouxo aumenta a possibilidade de formação de edema e, por consequência, o aparecimento de outras manifestações pós-operatórias indesejáveis, como o trismo e a dor ${ }^{6}$.

Estudos comparando a efetividade do Laser de baixa intensidade (LBI) no controle da DPO na exodontia de terceiros molares inferiores inclusos (dor, edema e trismo) são importantes para avaliar a possibilidade dessa fototerapia ser usada como principal agente anti-inflamatório e analgésico, evitando assim efeitos adversos dos fármacos convencionalmente utilizados ${ }^{7}$.

O Laser (Light Amplification by Stimulated Emission of Radiation) é uma poderosa fonte de luz, que tem inúmeras aplicações em todos os campos da ciência, incluindo nas áreas da saúde como a Odontologia $^{8}$. Em relação à aplicação clínica, o Laser pode ser classificado em dois grandes grupos: Laser de alta potência ou cirúrgicos, apresentando efeitos térmicos, propriedades de corte, vaporização e hemostasia e o Laser de baixa potência ou terapêuticos, com propriedades analgésicas, anti-inflamatórias e de bioestimulação ${ }^{9}$.

O LBI apresenta grandes possibilidades de tratamentos em várias especialidades odontológicas, mostrando-se um método seguro, não farmacológico, que modula vários processos metabólicos através da absorção de energia pelos cromóforos. A partir desse fenômeno, há uma alteração da função mitocondrial e, consequentemente, da respiração celular com aumento da produção de ATP (Adenosina Trifosfato), que produz espécies reativas de oxigênio intracelular (ROS - Reactive Oxygen Species). Essas alterações resultam na proliferação de fibroblastos, síntese de colágeno, ajuste da resposta inflamatória, bem como melhora da angiogênese e reparo tecidual ${ }^{10}$.

Pesquisas citam a eficiência da laserterapia em vários modelos de estudos, atuando como um anti-inflamatório restaurador de tecidos, amenizando o uso de analgésicos, porém, a variedade de modelos de análise e a diversidade de dosimetria sugerem a realização de ensaios clínicos mais amplos e longitudinais com padronização nos parâmetros de irradiação, favorecendo assim, a reprodutibilidade e os reais benefícios dessa terapia ${ }^{11}$.

A prevenção e o controle da inflamação desencadeada pelo ato cirúrgico de terceiros molares inferiores inclusos são preocupações constantes dos profissionais ${ }^{12}$. Diante dos benefícios descritos sobre a laserterapia e tendo o conhecimento que a exodontia é um procedimento amplamente realizado e que pode gerar desconforto pós-operatório, este estudo objetivou avaliar os possíveis efeitos benéficos do uso coadjuvante do LBI no pós-operatório.

\section{MATERIAL E MÉTODO}

Este estudo foi submetido ao Comitê de Ética da Universidade Federal de Mato Grosso do Sul tendo sua aprovação sob o CAAE de № 07702219.4.0000.0021.

Participaram deste estudo 10 voluntários que foram triados em um Centro de Saúde Pública de Rondonópolis-MT, Brasil, operados no período matutino (entre 8 e 10 horas), em ambiente climatizado, em consultório privado, após a realização de exame radiográfico panorâmico. Os participantes não apresentavam condições de saúde locais ou sistêmicas que contra indicavam a intervenção cirúrgica.

Os critérios de inclusão usados foram: ambos os sexos, com idade entre 16 e 26 anos; em bom estado geral de saúde, estado físico ASA I (American Society of Anesthesiologists), ou seja, normal saudável, sem distúrbios fisiológicos, bioquímicos ou psiquiátricos; com indicação para exodontia dos terceiros molares inferiores inclusos esquerdo e direito similares em ambos os lados, em posição Classe III, segundo a classificação radiográfica de Pell e Gregory ${ }^{13}$.

Os critérios de exclusão foram: presença de doenças sistêmicas; uso de qualquer droga ou fármaco nos últimos 15 dias antes das cirurgias; história de hipersensibilidade a algum dos fármacos, substancias e materiais que serão utilizados no experimento; mulheres em gestação ou lactação e período menstrual na época das cirurgias; infecção local como pericoronarites, abscessos e periodontites; traumas ou fraturas na região; pacientes que necessitam de terapia ansiolítica pré-operatória; doenças associadas ao terceiro molar.

- Delineamento

Esse foi um estudo split mouth, duplo cego, randomizado. Os participantes da pesquisa receberam a mesma terapêutica farmacológica, porém, com dois tratamentos distintos em relação à laserterapia, em tempos diferentes, delineados da seguinte maneira: 1) Uma cápsula de nimesulida (100mg), 10 minutos após o término da cirurgia e a cada 12 horas, por 3 dias, e tratado com laserterapia logo após a anestesia e sutura com Laser vermelho $660 \mathrm{~nm}$, com uma dose de $3 \mathrm{~J}$ por 30 
segundos nestes dois tempos cirúrgicos nas faces vestibular e lingual da região operada, e no pós-operatório mediato de 24 e 72 horas. $O$ Laser infravermelho $808 \mathrm{~nm}$ com uma dose de 3J por 30 segundos também foi utilizado nos linfonodos submandibulares, submentuais e cervicais logo após a cirurgia e no pósoperatório de 24 e 72h, como indicação de drenagem dos marcadores inflamatórios (Grupo Laser - GL). 2) Uma cápsula de nimesulida (100mg), 10 minutos após o término da cirurgia e a cada 12 horas, por 3 dias, e não receberam a laserterapia (Grupo Controle - GC).

Todos os participantes receberam uma cartela com 10 comprimidos de dipirona sódica(500mg) como fármaco de resgate para uso em caso de dor, que foi utilizado com intervalo de $6 \mathrm{~h}$. Esse esquema posológico foi escolhido uma vez que, os voluntários já se encontravam sob efeito de anti-inflamatório com provável efeito analgésico.

O cirurgião responsável pelo procedimento cirúrgico não tinha conhecimento prévio se o Laser foi utilizado, assim como o participante também não. Para isso, o aparelho foi utilizado, por outro profissional, no modo desligado, se ausentando o cirurgião da sala no momento do emprego ou não do Laser. Para que 0 paciente não soubesse quando 0 aparelho estava ou não funcionando, óculos de proteção escuro foi usado, além do seu uso ser condição para aplicação do Laser. A análise dos resultados foi realizada também por profissional sem conhecimento dos protocolos utilizados.

\section{- Procedimentos cirúrgicos}

A antissepsia extraoral foi realizada com solução aquosa de digluconato de clorexedina a 2\% (Vitale, Farmácia de Manipulação) e a intraoral feita por bochecho, durante um minuto, com solução aquosa de digluconato de clorexidina a $0,12 \%$ (Periogard(B).

Foi realizada anestesia tópica utilizando lidocaína a 5\% (Xylocaína Pomada - Astra Zeneca Produtos Farmacêuticos, Ltda.) e, em seguida, a anestesia dos nervos alveolar inferior e lingual com complementação anestésica para o nervo bucal, utilizando uma seringa do tipo carpule com refluxo (Duflex®-Brasil) com agulha gengival longa 27G (Unoject@ - DFL - Brasil). A complementação para o nervo bucal foi feita de forma associada, através de uma anestesia terminal infiltrativa subperiostal na região vestibular do fórnix, para possibilitar melhor descolamento do retalho, hemostasia adequada e dessensibilização do nervo bucal.

Para a anestesia foi padronizado um volume de $4,5 \mathrm{~mL}$ (equivalente ao contido em dois tubetes e meio) da solução anestésica de cloridrato de mepivacaína a $2 \%$ com epinefrina 1:100.000 (Mepivalem® - Dentsply - Brasil).

- Intervenção cirúrgica

As exodontias foram realizadas pelo mesmo operador, no período da manhã, seguindo o protocolo abaixo:

1 - Incisão mucoperiostal linear, com auxílio de uma lâmina de bisturi número 15 (MedBlade ${ }^{\circledR}$ Brasil) montada em cabo de bisturi número 3 na região distal do segundo molar inferior associada a incisão relaxante vestibular na região mesial do segundo molar (retalho em L);

2 - Na sequência, foram realizadas a sindesmotomia e descolamento mucoperiostal com destaca-periósteo do tipo Molt (Quinelato $\circledR$ - Brasil). Foi utilizado o afastador de Minessota (Quinelato® - Brasil) para exposição do campo operatório;

3 - Osteotomia e odontosecção por meio de fresa tronco-cônica $n^{\circ} 702$ (KG Sorensen $®$ Brasil) montada em caneta de alta rotação Magno 604 (Kavo® - Brasil);

4 - Irrigação abundante com solução fisiológica (SF) estérila 0,9\% (Sanobiol® - Brasil);

5 - Exodontia com auxílio de extratores tipo Seldin ( $n^{\circ} 2,1 R$ ou 1L) (Quinelato® - Brasil);

6 - Curetagem, inspeção cuidadosa, remoção de espículas ósseas com lima para osso (Quinelato $®$ - Brasil) e irrigação abundante da loja cirúrgica com SF;

7 - Coaptação das bordas do retalho, seguida de sutura simples com pontos separados, empregando fios de Nylon 4.0 Ethycon (Jonhson\&Jonhson®).

Todos os participantes receberam orientações pós-operatórias.

\section{- Avaliação da dor pós-operatória}

Para a avaliação da presença e intensidade da DPO, foi empregada uma ficha, identificando o participante com um código numeral e o protocolo utilizado.

Esta mesma ficha contém uma escala analógica visual (EAV) modificada ${ }^{14}$ na qual os pacientes foram orientados a anotar a presença e o grau de dor manifestada durante as $24 \mathrm{~h}$ após a cirurgia. A EAV é uma linha de $10 \mathrm{~cm}$, onde dependendo do local que o paciente faz a marcação, a dor é classificada em ausente, leve, moderada e intensa (Tabela 1).

Esta tabela foi usada para quantificar as anotações dos pacientes, ele não foi demonstrado ao participante da pesquisa, para não influenciar na sua marcação.

Nos casos em que a intensidade da dor obrigou o uso de fármaco de resgate, os voluntários foram instruídos a tomar um comprimido de dipirona sódica $(500 \mathrm{mg})$ via oral, sendo que foram fornecidos 10 comprimidos 
para cada voluntário, de forma que fosse anotada na ficha, a hora de ingestão deste medicamento, assim como a experiência de dor nesse momento ${ }^{15}$. Caso a dor persistisse, esse procedimento poderia ser repetido o quanto fosse necessário, obedecendo um intervalo mínimo de $6 \mathrm{~h}$ entre as doses. As anotações foram feitas, com 2, 6, 12, 24h de pósoperatório.

Tabela 1. Valores para mensurar e classificar a dor pós-operatória na escala analógica visual

\begin{tabular}{l|l}
\hline DOR & Medidas da EAV \\
\hline Sem & Zero a $4 \mathbf{~ m m}$ \\
\hline Leve & $\mathbf{5}$ a $44 \mathbf{~ m m}$ \\
\hline Moderada & $\mathbf{4 5}$ a $\mathbf{7 4} \mathbf{~ m m}$ \\
\hline Intensa & $\mathbf{7 5}$ a $100 \mathrm{~mm}$ \\
\hline 0
\end{tabular}

- Avaliação da presença de infecção no local operado

A infecção no pós-operatório foi investigada por meio de exame físico, sendo caracterizada pela presença de dor aguda, febre, limitação da abertura bucal, linfadenite e exsudato purulento. Caso esses sinais e sintomas se manifestassem depois de $72 \mathrm{~h}$ do término da cirurgia, seria realizado tratamento para a devida infecção. Caso essa complicação ocorresse, o voluntário seria retirado do estudo.

- Avaliação da presença de efeitos adversos do fármaco empregado

Ainda, na mesma ficha de avaliação de dor, foi questionado ao paciente a ocorrência ou não de efeitos adversos relacionados aos fármacos empregados na presente pesquisa, verificando a presença de sonolência, tontura, relaxamento muscular, problemas gastrointestinais (acidez estomacal, náuseas, vômito) e sinais de alergia (urticária, coceira) dentre outros sinais e sintomas.

- Método de avaliação do edema posoperatório

No pré-operatório foi avaliado o contorno facial do paciente através da técnica descrita por Markovic e Todorovic $^{16}$. Esta técnica (Figura 1) consiste na medida da distância entre a ponta do queixo até o bordo inferior do lobo auricular (PQLA). Estas medidas foram anotadas em fichas individuais.

O edema foi avaliado no pós-operatório de 24 e 72h e medido em centímetros. A fim de garantir a precisão das medidas, foram realizadas pequenas marcas nos pontos já descritos (parte inferior do lobo auricular e linha média na ponta do queixo) com tinta tipo "henna", a qual permaneceu por aproximadamente 15 dias.

- Avaliação da limitação da abertura bucal

Para avaliação da abertura bucal foi considerada a abertura máxima pré-operatória, medida em milímetros, com um paquímetro digital (PROFIELD $\circledast$, Alemanha) na borda incisal, entre os incisivos centrais superiores e inferiores direitos (Figura 2). Esse procedimento foi realizado também após 24 e $72 \mathrm{~h}$ de posoperatório, sendo considerada a diferença entre os valores pré e pós-operatórios como valor da limitação de abertura bucal em milímetros.

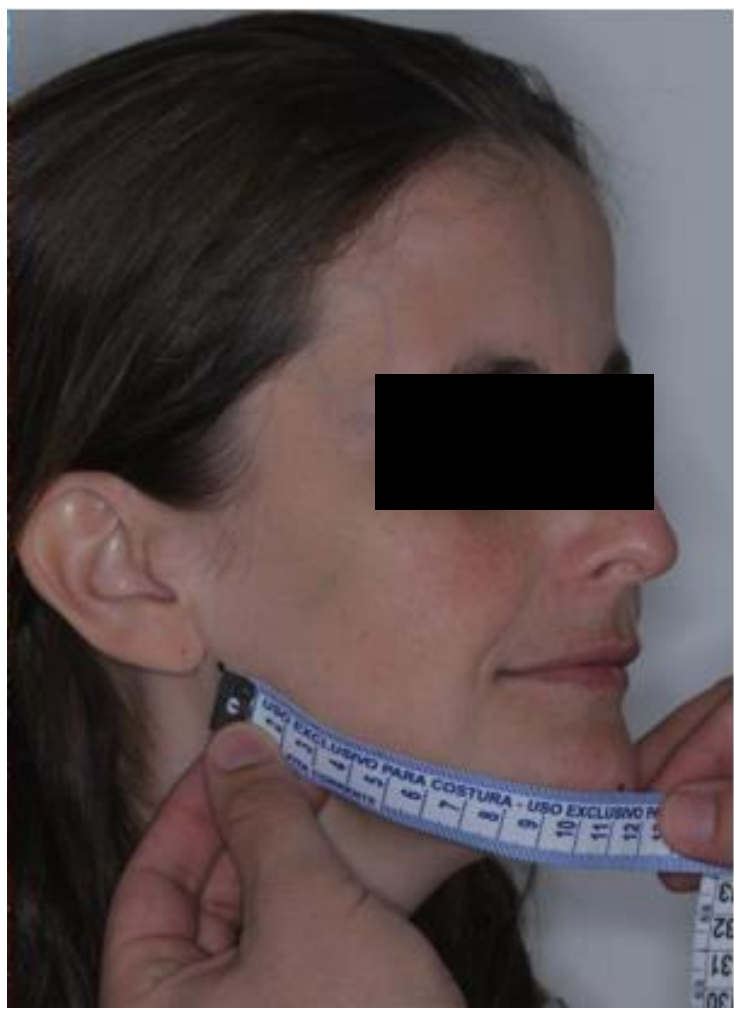

Figura 1: Método de avaliação do edema.

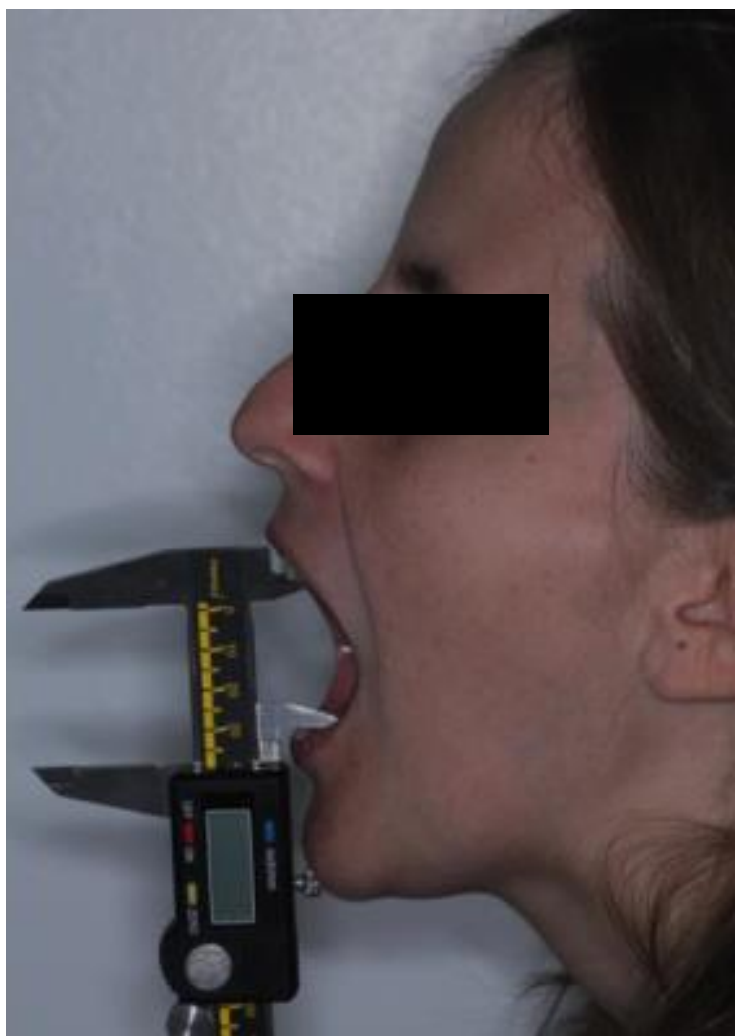

Figura 2: Método de avaliação da abertura bucal 


\section{- Análise estatística}

A comparação entre os lados de ambos os grupos (GL e $\mathrm{GC}$ ), em relação às variáveis nível de dor como avaliada pela EAV, distância da ponta do queixo até a parte inferior do lobo auricular e abertura da boca, em cada momento de análise, foi realizada por meio do teste $t$ de Student pareado. Já, a comparação entre os momentos de análise, em cada lado experimental, em relação às mesmas variáveis, foi realizada por meio do teste ANOVA de uma via de medidas repetitivas, seguido pelo pósteste de Tukey, quando pertinente. Os demais resultados deste estudo foram apresentados na forma de estatística descritiva ou na forma de tabelas e figuras. A análise estatística foi realizada por meio do programa estatístico SigmaPlot, versão 12.5 , considerando um nível de significância de $5 \%{ }^{17}$.

\section{RESULTADOS}

Neste estudo foram avaliados 10 pacientes, sendo 7 do sexo feminino e 3 do sexo masculino. A idade variou entre 16 e 26 anos, com média de $20,40 \pm 1,20$ anos (médiaterro padrão da média).

$\mathrm{Na}$ Tabela 2 estão apresentados os resultados referentes ao nível de dor como avaliada pela EAV, a distância ponta do queixo até a parte inferior do lobo auricular e a abertura da boca, de acordo com o lado experimental, em cada momento de análise, em relação à cirurgia.

Tabela 2. Níveis de dor como avaliada pela escala analógica visual, a distância da ponta do queixo até a parte inferior do lobo auricular e a abertura da boca, de acordo com o lado experimental, em cada momento de análise, em relação à cirurgia

\begin{tabular}{|c|c|c|c|}
\hline \multirow{2}{*}{$\begin{array}{l}\text { Variáveis/ } \\
\text { momentos }\end{array}$} & \multirow[b]{2}{*}{ Grupo controle } & \multirow[b]{2}{*}{ Grupo Laser } & \multirow{2}{*}{ Valor de $p$} \\
\hline & & & \\
\hline \multicolumn{4}{|c|}{ Nível de dor avaliada pela EAV (zero a $10 \mathrm{~cm}$ ) } \\
\hline Após 2 horas & $2,10 \pm 0,38 \mathrm{Aa}$ & $0,00 \pm 0,00 B a$ & $<0,001$ \\
\hline Após 6 horas & $2,10 \pm 0,50 A a$ & $0,30 \pm 0,21 \mathrm{Ba}$ & 0,003 \\
\hline Após 12 horas & $3,40 \pm 0,27 \mathrm{Aa}$ & $0,60 \pm 0,31 \mathrm{Ba}$ & $<0,001$ \\
\hline Após 24 horas & $2,00 \pm 0,62 \mathrm{Aa}$ & $0,00 \pm 0,00 B a$ & 0,010 \\
\hline Valor de $\mathrm{p}$ & 0,162 & 0,115 & \\
\hline \multicolumn{4}{|c|}{ Distância ponta do queixo até a parte inferior do lobo auricular $(\mathrm{cm})$} \\
\hline Pré-operatório & $13,05 \pm 0,26 \mathrm{Ab}$ & $13,05 \pm 0,26 \mathrm{Aa}$ & 1,000 \\
\hline Após 24 horas & $13,65 \pm 0,28 \mathrm{Ab}$ & $13,05 \pm 0,26 \mathrm{Ba}$ & 0,005 \\
\hline Após 72 horas & $13,91 \pm 0,32 \mathrm{Aa}$ & $13,05 \pm 0,26 \mathrm{Ba}$ & 0,009 \\
\hline Valor de $\mathrm{p}$ & 0,001 & 1,000 & \\
\hline \multicolumn{4}{|c|}{ Abertura bucal (mm) } \\
\hline Pré-operatório & $48,36 \pm 1,48 \mathrm{Aa}$ & $48,45 \pm 1,45 \mathrm{Aa}$ & 0,204 \\
\hline Após 24 horas & $26,09 \pm 1,02 \mathrm{Bb}$ & $35,32 \pm 1,90 \mathrm{Ab}$ & $<0,001$ \\
\hline Após 72 horas & $26,31 \pm 1,17 \mathrm{Bb}$ & $37,36 \pm 1,82 \mathrm{Ab}$ & $<0,001$ \\
\hline Valor de $\mathrm{p}$ & $<0,001$ & $<0,001$ & \\
\hline
\end{tabular}

Os resultados estão apresentados em media terro padrão da média. Valor de $p$ no teste $t$ de Student pareado (comparação entre lados) ou no teste ANOVA de uma via de medidas repetitivas (comparação entre momentos). Letras maiúsculas diferentes na linha indicam diferenças entre os lados controle e laser (teste $t$ de Student pareado, $\mathrm{p}<0,05$ ).
Letras minúsculas diferentes na coluna indicam diferença significativa entre os momentos de análise (pós-teste de Tukey, $p<0,05$ ).

Em relação ao nível de dor apresentado pelos pacientes, houve diferença significativa entre os lados experimentais (GCversusGL) em todos os momentos de análise (teste $t$ de Student pareado, valor de $\mathrm{p}$ variando entre $<0,001$ e 0,010 ), sendo que o nível da dor no lado em que foi aplicado o Laser foi sempre menor do que aquele no lado controle. Por outro lado, não houve diferença significativa entre os momentos de análise, em relação ao nível de dor, tanto no lado controle como no lado em que o laser foi aplicado (teste ANOVA de uma via de medidas repetitivas, lado controle: $p=0,162$; lado tratado com laser: $p=0,115)$. Estes resultados também estão ilustrados na Figura 3.

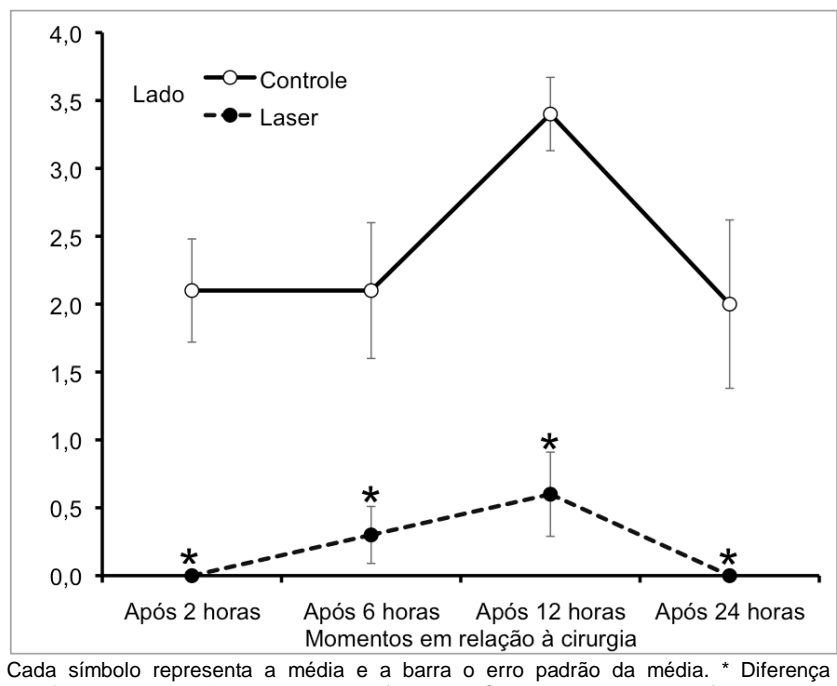
significativa em relação ao lado controle (teste $t$ de Student pareado, $p<0,05$ ).

Figura 3: Nível de dor, mensurado pela escala analógica visual (zero a $10 \mathrm{~cm}$ ), de acordo com o lado experimental (GCversusGL), em cada momento de análise do estudo, em relação à cirurgia.

Quanto à distância da ponta do queixo até a parte inferior do lobo auricular, tanto no momento após $24 \mathrm{~h}$ quanto no momento após $72 \mathrm{~h}$, a distância da ponta do queixo até a parte inferior do lobo auricular, no lado que foi aplicado o laser, foi significativamente menor do que aquela observada no lado controle (24h: $p=0,005 ;$ 72h: $p=0,009)$, indicando edema menor no $G L$ dois momentos. Quando comparados os momentos de análise, no lado controle houve diferença entre os momentos de análise, em relação à distância da ponta do queixo até a parte inferior do lobo auricular (teste ANOVA de uma via de medidas repetitivas, $p=0,001$ ), sendo que após $72 \mathrm{~h}$ ela foi maior do que aquela observada nos momentos pré-operatório e após $24 \mathrm{~h}$ (pós-teste de Tukey, $p<0,05)$ indicando um aumento do edema após $72 \mathrm{~h}$. Por outro lado, não houve diferença entre os momentos de análise, em relação à distância da ponta do queixo até a 
parte inferior do lobo auricular no lado tratado com Laser $(p=1,000)$. Estes resultados também estão ilustrados na Figura 4. Para a abertura bucal, tanto no momento após $24 \mathrm{~h}$ quanto no momento após $72 \mathrm{~h}$, a abertura bucal, no lado que foi aplicado o Laser, foi significativamente maior do que aquela observada no lado controle (24 e 72h: $p<0,001$ ). Quando comparados os momentos de análise, tanto no lado controle como no lado tratado com laser, houve diferença entre os momentos de análise, em relação à abertura bucal (teste ANOVA de uma via de medidas repetitivas $p<0,001)$ sendo que em ambos os lados, a abertura da boca nos momentos após 24 e após $72 \mathrm{~h}$ foi menor do que aquela observada no momento pré-operatório (pós-teste de Tukey, $\mathrm{p}<0,05)$. Estes resultados também estão ilustrados na Figura 5.

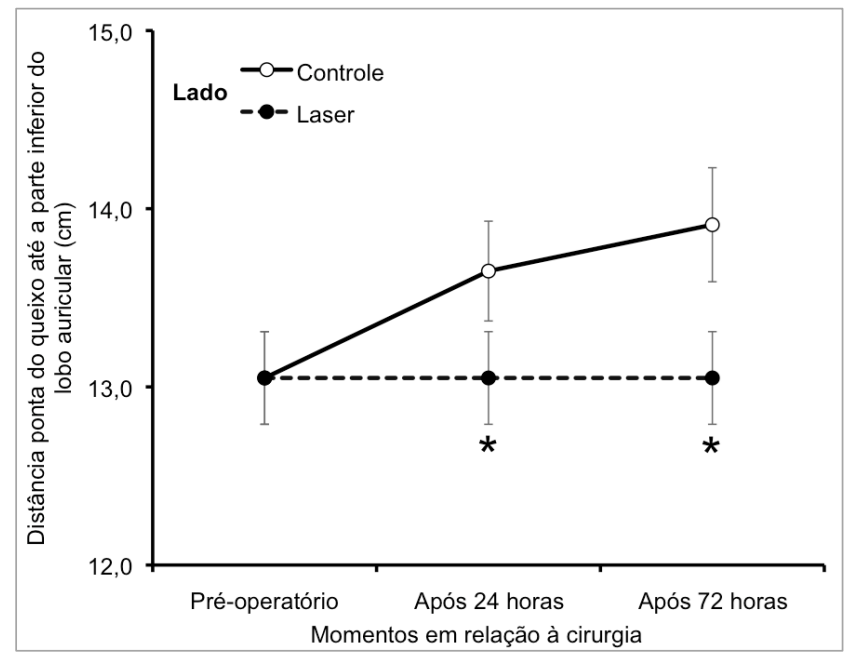

Cada símbolo representa a média e a barra o erro padrão da média. * Diferença significativa em relação ao lado controle (teste t de Student pareado, $\mathrm{p}<0,05$ ).

Figura 4: Distância da ponta do queixo até a parte inferior do lobo auricular, de acordo com o lado experimental (Controle versus Laser), em cada momento de análise do estudo, em relação à cirurgia.

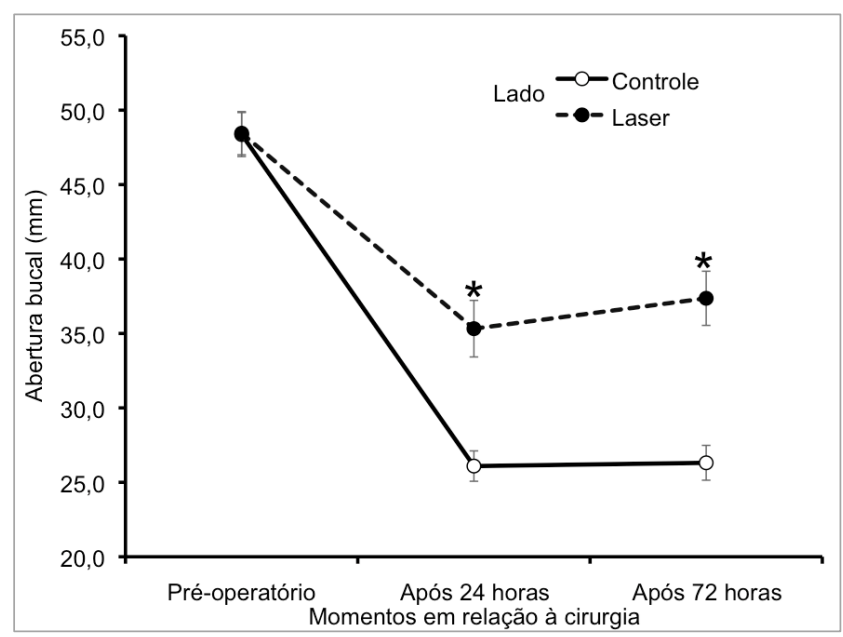

Cada símbolo representa a média e a barra o erro padrão da média. * Diferença significativa em relação ao lado controle (teste $t$ de Student pareado, $p<0,05$ ).

Figura 5: Abertura bucal, de acordo com o lado experimental (Controle versus Laser), em cada momento de análise do estudo, em relação à cirurgia.
DISCUSSÃO

A exodontia de terceiros molares inclusos está indicada como prevenção de doença periodontal, pericoronarite, de processos cariosos, reabsorção radicular, futuras fraturas ósseas do tipo patológicas, como fator profilático de cistos de natureza odontogênica, além de favorecimento de espaços para a movimentação ortodôntica ${ }^{18}$. Várias alternativas terapêuticas são estudadas com o objetivo de oferecer ao organismo uma melhor capacidade de regeneração tecidual, minimização da dor e diminuição de edemas, a partir da bioestimulação celular ${ }^{6}$.

O LBI é utilizado como agente terapêutico após o tratamento convencional, mostrando propriedades antiinflamatórias, analgésicas e de aceleração da cicatrização de feridas, assim pode propiciar um pós-operatório mais confortável ao paciente, diminuindo a dor pós operatória, possibilitando a redução do uso de medicamentos, por apresentar propriedades que produzem aumento da proliferação da atividade celular, aumento da produção de colágeno e da síntese de DNA, modulação da produção dos fatores de crescimento e redução na produção de prostaglandinas ${ }^{19-22}$.

Em estudo com 120 pacientes obteve diferenças entre os tratamentos propostos, mostrando desta forma que esta metodologia é capaz de detectar diferenças de aumento volumétrico na região mandibular ${ }^{16}$. A mensuração do edema, utilizada nesta pesquisa, permitiu observar com precisão um aumento significativo a partir de 24 até 72 h após remoção dos terceiros molares. Esses resultados concordam com os dados de estudos que observaram também a presença do edema nos tempos de 48 e 72 horas após o procedimento cirúrgico ${ }^{23,24}$. Na presente investigação houve diferença significativa na diminuição do edema no lado que foi aplicado o laser. Esta diminuição pode ser explicada por uma ação do laser diretamente na célula, produzindo um efeito primário ou imediato aumentando 0 metabolismo celular, aumentando a síntese de endorfinas e diminuindo a liberação de transmissores nociceptivos, como a serotonina e a bradicinina. Também há um efeito secundário ou indireto, aumentando o fluxo sanguíneo e a drenagem linfática, e por fim, há a instauração de efeitos terapêuticos gerais ou efeitos tardios, sendo que, clinicamente, observa-se a ativação do sistema imunológico ${ }^{25}$.

Para a avaliação da dor, medida pela escala EAV modificada $^{15}$ a mesma foi 
mensurada por $24 \mathrm{~h}^{26}$, pois, a dor ocorre usualmente $12 \mathrm{~h}$ após a cirurgia, com intensidade máxima após $6 \mathrm{~h}^{27}$. $\mathrm{Na}$ análise de nível da dor, apresentado pelos pacientes, foi observado uma diferença significativa entre os lados experimentais (Controle versus Laser). Em todos os momentos, o nível da dor no lado em que foi aplicado o laser foi menor do que aquele do lado controle. Essas ações analgésicas acontecem por manutenção do potencial transmembrana, evitando a despolarização, por meio do aumento da síntese de ATP, essa energia é usada para expulsar os íons sódio do interior da célula dificultando a transmissão do estímulo doloroso local $^{12,28}$.

Considerando as principais variáveis desse estudo, a limitação de abertura bucal, conhecida como trismo, também foi analisada a medida máxima pré-operatória registrada em milímetros. Esse procedimento foi realizado também após 24 e $72 \mathrm{~h}$ de pos-operatório, sendo considerada a diferença entre os valores pré e pós-operatórios como valor da limitação de abertura bucal ${ }^{12,22,29}$, sendo que houve diferença significativa na melhora de abertura bucal nos pacientes tratados com LBI. Isto pode ser explicado em razão do $\mathrm{LBI}$ auxiliar em disfunções musculares por possuir efeitos analgésicos, anti-inflamatórios e bioestimulantes. Esta condição, quando não tratada adequadamente, dificulta os movimentos mandibulares, prejudica a alimentação, interfere na higiene oral, restringe 0 acesso para procedimentos orais, e pode afetar até mesmo a fala e a aparência facial. Em casos mais graves, o trismo pode afetar a função pulmonar de indivíduos respiradores bucais ${ }^{4}$.

A terapia com LBI destaca, entre seus principais efeitos, a reparação tecidual, benefícios no caráter analgésico, antiinflamatório e cicatrizante ${ }^{21,30}$, inibindo a produção de prostaglandinas por ação da enzima cicloxigenase sobre 0 ácido araquidônico, além de ação na microcirculação local e na ativação do sistema linfático. Portanto, sugere-se que esta aplicação seja feita nos principais linfonodos responsáveis pela drenagem, objetivando assim acelerar tal processo ${ }^{20,31-32}$. Isso foi confirmado nos resultados deste estudo quando se analisou a variável do edema no lado que foi aplicado o $\mathrm{LBI}$, que se mostrou significantemente menor do que aquela observada no lado controle.

CONCLUSÃO

A terapia com LBI para controle no nível de dor, edema e trismo após a exodontia de terceiros molares inferiores inclusos, proporcionou efeitos benéficos ao paciente. Entretanto, são necessários estudos adicionais, especialmente na busca por um protocolo padrão sobre parâmetros de irradiação a ser empregada, tempo de utilização e locais de aplicação.

\section{AGRADECIMENTOS}

O presente trabalho foi realizado com apoio da Fundação Universidade Federal de Mato Grosso do Sul - UFMS/MEC - Brasil

\section{REFERÊNCIAS}

1. Rosa FM, Escobar CA, Brusco LC. Parestesia dos nervos alveolar inferior e lingual pós cirurgia de terceiros molares. RGO. 2007;55(3):291-5.

2. Bui $\mathrm{CH}$, Seldin EB, Dodson TB. Types, frequencies, and risk factors for complications after third molar extraction. J Oral Maxillofac Surg. 2003;61(12):1379-89.

3. Araujo OC, Agostinho CN, Marino LM, Rabêlo LR, Bastos EG, Silva VC. Incidência dos acidentes e complicaçõesemcirurgias de terceirosmolares. Rev Odontol UNESP. 2011;40(6):290-5.

4. Matos, AA. Limitação de aberturabucalapós exodontias-revisão de literatura. 2015. 21fls. Trabalho de conclusão de curso (Graduação em Odontologia) - Universidade Estadual de Londrina. Londrina, 2015.

5. Felix VB. Implante de alveosan e de anaseptil pó associado ao eugenol e alveolus dentais após a exodontia. Estudo microscópico em ratos. Marília, Dissertação - Faculdade de Ciências Odontológicas da Universidade de Marília. 2006.

6. Maluf AP, Ughini GC, Maluf RP, Pagnoncelli RM. Utilização de laser terapêutico em exodontia de terceiros molares inferiores. RGO. 2006;54(2):182-4.

7. Prockt AP, Takahashi A,Pagnoncelli RM. Uso de terapia com laser de baixa intensidade na cirurgia bucomaxilofacial. Rev Port Estomatol Cir Maxilofac. 2008;49(4):247-55.

8. Srivastava VK,Mahajan S. Diode lasers: a magical wand to an orthodontics practice. Indian J Dent Res. 2014:25(1):78-82

9. Barros FC, Antunes SA, Figueredo CM, Fischer $R G$. Laser de baixa intensidade na cicatrização periodontal. R Cir Méd Biol. 2008;7(1):85-9.

10. Sant'Anna EF, Araújo MTS, Nojima LI, Cunha $A C D$, Silveira BLD, Marquezan M. Highintensity laser application in orthodontics. Dental Press J Orthod. 2017;22(6):99-109.

11. Mileto TN, Azambuja FG. Eficácia do laser de baixa intensidade no pós-operatório em exodontia de terceiros molares. RGO. 2017; 65(1):13-9. 
12. Agra PA, Nascimento JS, Fontes KB, Picciani $\mathrm{BL}$, Monteiro MC. Os efeitos do laser de baixa potência no tratamento de mucosite oral associada a quimioterapia. Rev Bras Odontol. 2018;75(Suppl 1):35

13.Pell GJ, GREGORY BT. Impacted mandibular third molars classification and modified technique for removal. Dental Dig. 1933; 39:330-38.

14. Jensen MP, Karoly P, Braver S. The measurement of clinical pain intensity: a comparison of six methods. Pain. 1986;27(1):117-26.

15. Girotto MA. Estudo comparativo de doisprotocolosfarmacológicosem exodontias de terceiros molares mandibulares retidos (tese). Piracicaba: FOP/UNICAMP; 2003.184p.

16. Markovic A, Todorovic LJ. Effectiveness of dexamethasone and low-power laser in minimizing edema after third molar surgery: a clinical trial. Int $\mathrm{J}$ Oral Maxillofac Surg. 2007;36(2):226-9.

17. Rowe P. Essential statistics for the pharmaceutical sciences. Chichester, England: John Wiley \& Sons Ltda; 2007.

18. Marsal JM, Cama RR, Gonçalves CT, Barroso FV, Carlos G, Motta RH. Avaliação dos efeitos de duas formulações de dexametasona em cirurgias de terceiros molares inclusos. Rev Dor. 2014;15(3):163-8.

19. Lopes LA. Análise in vitro da proliferação celular de fibroblastos de gengiva humana tratados com laser de baixa intensidade utilizando diferentes parâmetros de irradiação (tese). São Carlos: Universidade de São Paulo - Escola de Engenharia de São Carlos. 2003.

20. Damante C, Marques M, De Micheli G. Terapia com laser em baixa intensidade na cicatrização de feridas - revisão de literatura. RevFac Odontol.2008;13(3):88-93.

21. Lins RD, Dantas EM, Lucena CR, Catão $M H$, Granville-Garcia AF, Carvalho Neto LG. Efeitos bioestimulantes do laser de baixa potência no processo de reparo. An Bras Dermatol. 2010;85(6):849-55.

22. Laureano Filho JR, Maurette PE, Allais M, Cotinho M, Fernandes C. Clinical comparative study if the effectiveness of two dosages of dexamethasone to control postoperative swelling, trismus and pain after the surgical extraction of mandibular impacted third molars. Med Oral Patol Oral Cir Bucal. 2008:13(2):E129-32.

23. Laureano Filho JR, Camargo IB, Firmo AC, Oliveira e Silva ED. A influência do laser de baixa intensidade na redução de edema, dor e trismo no pós-operatório de cirurgia de terceiros molares inferiores inclusos: resultado preliminar com 13 casos. Rev Cir Traumatol Buco-Maxilo-Fac. 2008;8(1):47-56.
24.De Menezes SA, Cury PR. Efficacy of nimesulide versus meloxicam in the control of pain, swelling and trismus following extraction of impacted lower third molar. Int $\mathrm{J}$ Oral Maxillofac Surg. 2010;39(6):580-4.

25. Atihe MM. Redução de Processo inflamatório com aplicação de laser de arseneto de gálio alumínio em pós-operatório de exodontias de terceiros molares inferiores inclusos ou semiinclusos. 2002, 37f. Dissertação (Mestrado em laser em Odontologia) - IPEN, Universidade de São Paulo, 2002.

26. Bamgbose BO, Akinwande JA, Adeyemo WL, Ladeinde AL, Arotiba GT, Ogunlewe MO. Effects of co-administered dexamethasone and diclofenac potassium on pain, swelling and trismus following third molar surgery. Head Face Med. 2005;1(11): 1-6.

27. Seymour RA, Meechan JG, Blair GS. An investigationinto post-operative pain after third molar surgery under local analgesia. $\mathrm{Br} \mathrm{J}$ Oral Maxillofac Surg. 1985;23(6):410-8.

28. Chow RT, Barnsley L. Systematic review of the literature of low-level laser therapy (LLLT) in the management of neck pain. Lasers Surg Med. 2005;37(1):46-52.

29. Batista VO, Meireles GC, Duarte SF, Carneiro JB. Laserterapia como alternativa terapêutica à nimesulida pós-cirurgias de terceiros molares impactados: relato de caso. Interscientia. 2013;1(1):66-79.

30. Lizarelli RFZ. Reabilitação BiofotônicaOrofacial: Fundamentos e protocolos Clínicos. São Carlos: Compacta Gráfica e Editora; 2018.

31. Henriques AC, Cazal C, Castro JF. Ação da laserterapia no processo de proliferação e diferenciação celular. Revisão de literatura. Rev Col Bras Cir. 2010:37(4):295-302.

32. Garcez SG, Ribeiro MS, Nunez SC. Laser de baixa potência: princípios básicos e aplicações clínicas na odontologia. Editora Elsevier; 2012.

\section{CONFLITO DE INTERESSES}

Os autores declaram não haver conflitos de interesse

\section{AUTOR PARA CORRESPONDÊNCIA}

\section{Ellen Cristina Gaetti Jardim}

Universidade Federal de Mato Grosso do Sul - UFMS

Cidade Universitária, s/n Universitário

79070-900 Campo Grande - MS, Brasil

(67) 3345-7682

ellen.jardim@ufms.br 